\title{
Medicinal plants used by women from Agnalazaha littoral forest (Southeastern Madagascar)
}

\author{
Mendrika Razafindraibe ${ }^{1 *}$, Alyse R Kuhlman³, Harison Rabarison', Vonjison Rakotoarimanana', \\ Charlotte Rajeriarison ${ }^{1}$, Nivo Rakotoarivelo ${ }^{1,2}$, Tabita Randrianarivony ${ }^{1,2}$, Fortunat Rakotoarivony ${ }^{2}$, Reza Ludovic ${ }^{2}$, \\ Armand Randrianasolo ${ }^{3}$ and Rainer W Bussmann ${ }^{3}$
}

\begin{abstract}
Background: The country of Madagascar is renowned for its high level of biodiversity and endemism, as well as the overwhelming pressures and threats placed on the natural resources by a growing population and climate change. Traditional medicine plays an important role in the daily lives of the Malagasy for various reasons including limited access to healthcare, limited markets and traditional values. The objective of this study was to assess the modern utitilization of the Agnalazaha Forest by the local population in Mahabo-Mananivo, Madagascar, for medicinal plants used by women, and to establish a list of medicinal plants used by women sourced from Agnalazaha Forest.
\end{abstract}

Methods: Ethnobotanical studies were conducted over a period of five months in 2010 to determine the diversity of medicinal plants used by women in the commune of Mahabo-Mananivo. In all, 498 people were interviewed, both male and female ranging age from 15 to over 60 years old.

Results: 152 medicinal plants used by local people were collected during the ethnobotanical studies. Among the recorded species, eight native species are widely used by women. These species are known for their therapeutic properties in treating placental apposition and complications during childbirth as well as tropical illnesses such as malaria, filariasis, and sexual diseases like gonorrhea and syphilis.

Conclusions: Littoral forests are rare ecosystems that are highly threatened on the island nation of Madagascar. Our investigation into the use of medicinal plants sourced from and around the Agnalazaha Forest by the women of Mahabo-Mananivo reinforces the need for this natural resource as a first line of health care for rural families.

Keywords: Medicinal plants, Madagascar, Littoral forest, Traditional medicine, Women's traditional knowledge

\section{Background}

Traditional medicine is a term used to describe the use of natural resources, often in concert with ritual and spirituality, to prevent, treat and heal human diseases and ailments [1]. While the use of plant species for healing dates back further than the written record, with evidence the Neanderthals practiced plant medicine [2], it is still being used by many in our modern era. Eighty (80) percent of the world's population depends on

\footnotetext{
* Correspondence: mendrika_razafindraibe@yahoo.fr

${ }^{1}$ Department of Plant Biology and Ecology, Faculty of Science, University of Antananarivo, BP 566, Antananarivo 101, Madagascar

Full list of author information is available at the end of the article
}

traditional medicine for the treatment of pain [3]. And in developing countries such as Madagascar medicinal plants remain a primary source of medical care [4] especially in very remote areas or in case of limited health resources.

Medicinal plant use in Madagascar has the added concern of biodiversity loss, environmental degradation, and sustainability. The island nation of Madagascar separated from Africa some 170 million years and the Indian subcontinent nearly 88 million years ago and the isolated flora and fauna have evolved with a high degree of microendemism [5]. Current floristic calculations indicate Madagascar houses between 12,000 and 14,000

\section{Biomed Central}

(C) 2013 Razafindraibe et al.; licensee BioMed Central Ltd. This is an open access article distributed under the terms of the Creative Commons Attribution License (http://creativecommons.org/licenses/by/2.0), which permits unrestricted use, distribution, and reproduction in any medium, provided the original work is properly cited. 
vascular plant species, of which $90 \%$ are endemic [6] and $96 \%$ endemism in tree species [7]. However, the increasingly intense population growth has led to rapid deforestation as land is cleared for agricultural fields and for fuel [8]. Biodiversity loss, in general, has severe implications on environmental stability which in turn affects human health [9]. When biodiversity directly adds to the wellness of a community as a resource for medicine, biodiversity loss can have even deeper consequences as medicinal plant species are lost or are no longer available $[10,11]$.

Within Madagascar, one of the most threatened ecosystems is the littoral forest [12]. Although the littoral forests of Madagascar once stretched 1600-km along the eastern coast as one single biological corridor, there is only $10 \%$ of the original forest remaining [13]. One such littoral forest, the Agnalazaha Forest, is located in the rural commune of Mahabo-Mananivo, $750 \mathrm{~km}$ southeast of the capitol city of Antananarivo. Approximately $72.3 \%$ of the flora of Agnalazaha is endemic to Madagascar [14].

The villages of Mahabo-Mananivo source timber and non-timber forest products from Agnalazaha Forest littoral forest. Furthermore, the community of MahaboMananivo still practice and often prefers traditional medicine, especially for common diseases and infectious diseases [15]. As is the case with most familial systems, the first line of healthcare decisions and action is often administered by female household members [16]. The purpose of this study was to assess the modern utilization of this forest by the local population with a focus on the plants known and utilized by women in their everyday care giving. We focused on the women for this study while a study on the use of medicinal plants by men was carried out simultaneously. At times men were present during the interview process and would add information about plants used by women which we allowed.

\section{Methods}

Research was coordinated by and supported in large part by the staff at the Missouri Botanical Garden MahaboMananivo Conservation research site. Field research was conducted over a period of five months (January - May) in 2010 with three field trips to the community. A ten day preliminary exploration was used to become familiar with the community and introduce ourselves, make contact with local officials and present the topic of our research. A hired local guide acted as our translator, introduced us to interview prospects and coordinated interview schedules. Consent was given by the tribal leaders, local government officials and by each individual we interviewed.

\section{Study site}

Agnalazaha Forest is located within the district of Farafangana, Atsimo Atsinanana region in southeastern Madagascar, in the Commune Rural Mahabo-Mananivo
(Figure 1). The National Road 12, a paved highway connecting Farafangana and Vangaindrano borders the forest to the west while the Indian Ocean borders it to the east. It is between $47^{\circ} 41^{\prime}$ and $47^{\circ} 45^{\prime} \mathrm{E}$, and $23^{\circ} 09^{\prime}$ and $23^{\circ} 14^{\prime} \mathrm{S}$ with an altitude of less than $50 \mathrm{~m}$ [14]. In 2003, it was measured that this coastal forest covered an area of 1,565 ha and represents approximately $17 \%$ land coverage of the rural area of the commune Mahabo-Mananivo. Agnalazaha Forest has the status of Forest Reserve under article number 129-SF/EF/CG since May 17, 1954, but has been under the management of the Missouri Botanical Garden (MBG) since 2002.

The southeast region of Madagascar is characterized as the eastern coastal plain and has a climate of high rainfall and high average temperature [17]. The Agnalazaha Forest experiences two seasons: the hot rainy season from December to April, and the cool season from May to November. The average annual rainfall in Agnalazaha Forest is $2,706 \mathrm{~mm}$. The average annual temperature varies between $21^{\circ} \mathrm{C}-24^{\circ} \mathrm{C}\left(69^{\circ} \mathrm{F}-75^{\circ} \mathrm{F}\right)$. According to the bioclimatic division of Madagascar, this region belongs to the humid tropics and part of the humid warm bioclimatic type [18]. Agnalazaha Forest is classifed as a littoral forest, characterized by an open canopy and sandy soils [12], seasonally flooded wooded swamps, open marshes with Nepenthes madagascariensis and Lepironia articulata, savannas, remnants of secondary forest on lateritic soils and reforestation forests of Eucalyptus robusta and Acacia mangeum. There are 275 species of plants in Agnalazaha Forest [14] distributed within 188 genera and 82 families. The site contains species belonging to three endemic families, Asteropeiaceae (2 species), Sarcolaenaceae (6 species) and Sphaerosepalaceae (1 species). Furthermore, 199 species present in Agnalazaha Forest are determined to be endemic to Madagascar (72.3\%).

An inventory of primates [19] conducted in Agnalazaha Forest identified four species of lemur all of which are considered to at least be threatened, including the critically endangered Eulemur albocollaris [20]. All are known to be hunted locally. A similar study identified seven species of endemic small mammals found in Agnalazaha Forest including Pteropus rufus, Hemicentetes semispinosus, Setifer setosus and Tenrec caudatus, Hova oryzorictes, all of which are also locally hunted [21].

\section{Surrounding community}

Commune Rural Mahabo-Mananivo surrounds Agnalazaha Forest to the west, north and south. There are 6,998 residents according to the 2009 census. Mahabo-Mananivo is primarily comprised of residents identifying with the Antesaka ethnic group, while Antefasy, Merina and Betsileo members have migrated to this area as well. The municipality of Mahabo-Mananivo consists of ten fokontany surrounding the Agnalazaha Forest; Mahabo, 


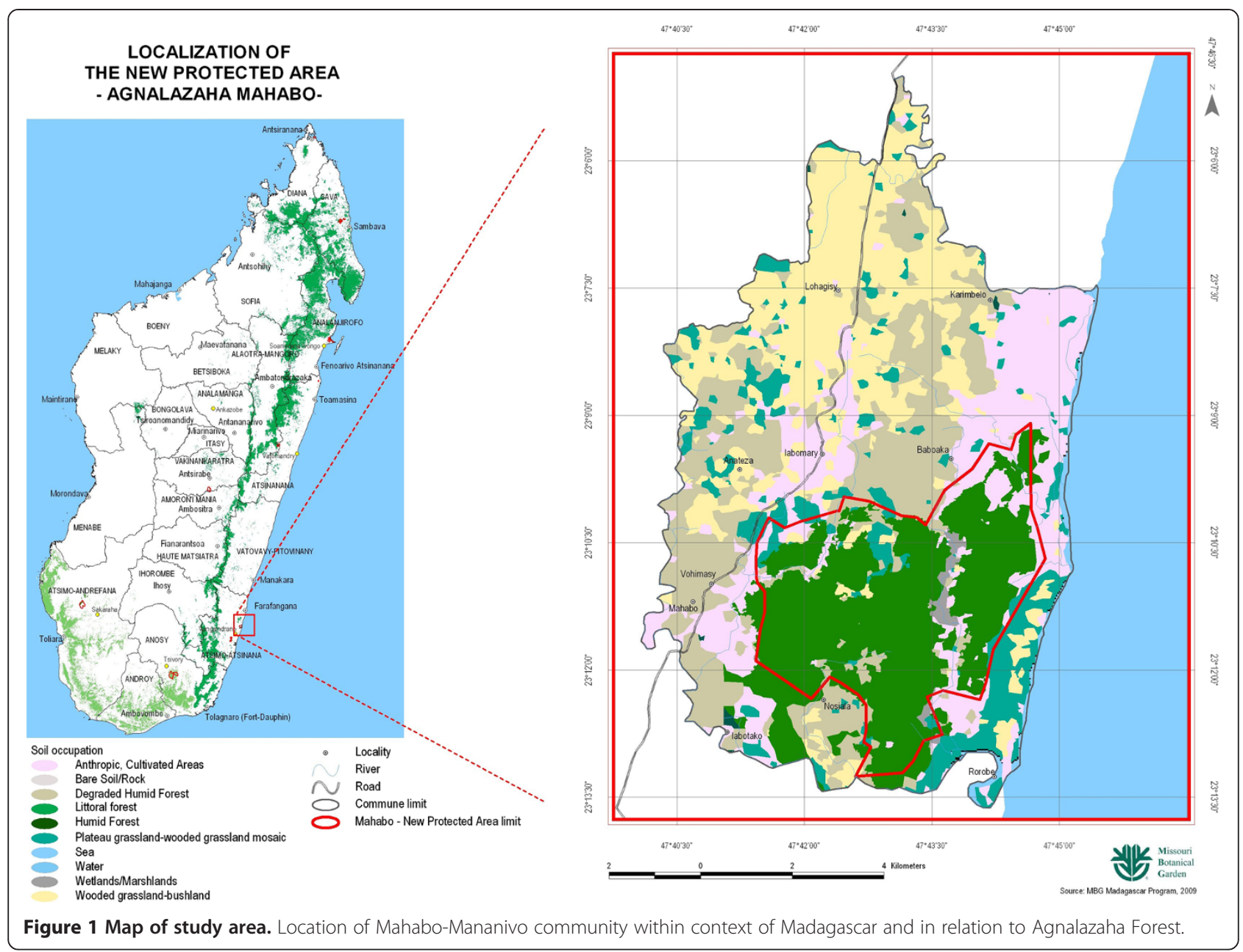

Vohimasy, Iabotako, Nosiala, Iambomary, Baboaka, Lohagisy, Karimbelo, Rorobe, and Agnateza. A "fokontany" is the smallest political distinction recognized by the government. It may compromise several small villages with an average 1,000 people [22]. MahaboManaivo is primarily an agriculture economy. Approximately $99.74 \%$ of the population is farmers. Rice fields dominate the landscape with cassava, yams, and manihot as supplementary crops. Additional income is sought through handicraft production, largely basketry weaving. The most popular species used for basketry is Lepironia articulate, Cyperus sp, and Pandanus sp. Monthly income is less than 80,000 Ariary (approximately USD\$35) for a majority of the population of Mahabo-Mananivo.

There is a relatively new community health center, built in 2009, in the fokontany Mahabo, located on Road 12. It has 2 rooms and accommodates one doctor and one nurse. However, a majority of the population of MahaboMananivo relies on medicinal plants to cure common diseases. To this end, local people consult traditional healers called ombiasy to be treated with medicinal plants.
Ombiasy can be distinguished into four different types of healers: tromba (spiritually possesed) healers, midwives, massage healers and premonition healers.

\section{Ethnobotanical surveys}

The Agnalazaha Forest provides the local population with firewood, timber for home construction, nontimber products and medicinal plants. In order to identify medicinal plants known to be used by and for women in the rural commune of Mahabo-Mananivo, inquiries on the therapeutic use of plants were conducted primarily with women and female healers, although some men were interviewed as well. Due to time limitations, not all fokontany were included in the study. Fokontany were selected using the following criteria: (a) proximity to Agnalazaha Forest (b) Distance to the health center located in Mahabo (c) presence of female healers in the village. Fokontany closest to Agnalazaha Forest were given priority. Field visits to each fokontany selected were scheduled so that the villages furthest from the forest were visited first. The interviews were structured as semi-direct interviews with open questions 
[23]. The interviews were conducted with both individuals and in group settings [24]. Interview questions were written with two different approaches, inquiry of plant specific use or through disease-specific and/or symptomatic description plant use. Questionnaires or survey forms were established, first on medicinal plants used by women and healers, then the socio-economic and cultural value for each species (Additional file 1).

Surveys focused on plants used in the treatment of common female diseases in the commune. They were conducted with traditional healers (ombiasy), birth attendants, women and men who know the medicinal plants used by and for women. The interviews were interspersed with forest walks with interview participants where species were identified by their vernacular names and photos were taken. Herbarium voucher were made and the identification of these species was then conducted in the national herbarium of Tsimbazaza (TAN).

\section{Results}

\section{Demographic variables}

In the community 498 people were surveyed, 301 (60.44\%) were women and 197 (39.56\%) were men and 90.56\% of those interviewed responded that they utilize medicinal plants. Table 1 compares the number of those who utilize medicinal plants with those who do not use medicinal plants for each age group, level of schooling, marital status and income level.

People aged 40 to 49 years have the highest frequency of use of medicinal plants at $98.29 \%$. This age group was followed by the 50 - 59 year old age bracket ( $96.15 \%)$, the 30 - 39 year old age bracket (94.59\%), 60 years and older bracket $(89.36 \%)$, the 20 - 29 years old bracket $(86.91 \%)$ and finally the youngest bracket, 15 - 19 years old at $45.73 \%$. We found that people at least 30 years old have increased knowledge in terms of medicinal plants, while lower knowledge levels occur in the younger age groups.

Furthermore, the data analysis shows that in the Commune of Mahabo-Mananavio, the majority of women $(65.90 \%)$ who use were interviewed are illiterate, with 96.34\% of them using medicinal plants. This high percentage is directly correlated with the fact that girls receive less education than boys. Persons with at least a primary school level of education made up $28 \%$ of our interviewees, and have a significant percentage of use of medicinal plants $(82.52 \%)$, while those with secondary level of education (4.8\% of our respondents) make little use of medicinal plants $(66.53 \%)$. This percentage decreases again and becomes less significant for those with a university level education (33\%).

Married people have a broad knowledge of medicinal plants with a percentage of $77.10 \%$, while persons listed as single use plants at a frequency of $21.10 \%$. Most of these

Table 1 Demographic information of the ethnobotanical interviewees

\begin{tabular}{|c|c|c|c|c|c|c|c|}
\hline & & $\begin{array}{r}\text { Number of } \\
\text { people } \\
\text { interviewed }\end{array}$ & Percentage of total & $\begin{array}{r}\text { Utilize } \\
\text { medicinal } \\
\text { plants (\#) }\end{array}$ & $\begin{array}{r}\text { Percentage } \\
\text { of total }\end{array}$ & $\begin{array}{r}\text { Do not utilize } \\
\text { medicinal } \\
\text { plants (\#) }\end{array}$ & $\begin{array}{r}\text { Percentage } \\
\text { of total }\end{array}$ \\
\hline \multirow[t]{2}{*}{ Gender } & Females & 301 & 60.44 & 280 & 93.02 & 21 & 6.98 \\
\hline & Men & 197 & 39.56 & 171 & 86.8 & 26 & 13.2 \\
\hline \multirow{6}{*}{ Age group } & [15-19] & 35 & 7.03 & 16 & 45.73 & 19 & 54.27 \\
\hline & [20-29] & 84 & 16.87 & 73 & 86.91 & 11 & 13.09 \\
\hline & [30-39] & 111 & 22.29 & 105 & 94.59 & 6 & 5.41 \\
\hline & {$[40-49]$} & 117 & 23.49 & 115 & 98.29 & 2 & 1.71 \\
\hline & {$[50-59]$} & 104 & 20.88 & 100 & 96.15 & 4 & 3.85 \\
\hline & {$[60+]$} & 47 & 9.44 & 42 & 89.36 & 5 & 10.64 \\
\hline Level of & Illiterate & 328 & 65.9 & 316 & 96.34 & 12 & 3.66 \\
\hline \multirow[t]{3}{*}{ Education } & Primary & 143 & 28.7 & 118 & 82.52 & 25 & 17.48 \\
\hline & Secondary & 24 & 4.8 & 16 & 66.53 & 8 & 33.47 \\
\hline & University & 3 & 0.6 & 1 & 33.33 & 2 & 66.67 \\
\hline \multirow[t]{3}{*}{ Marital status } & Single & 105 & 21.1 & 80 & 76.21 & 25 & 23.79 \\
\hline & married & 384 & 77.1 & 368 & 95.83 & 16 & 4.17 \\
\hline & widowed & 9 & 1.8 & 3 & 33.07 & 6 & 66.93 \\
\hline Household & $<80,000$ & 371 & 74.5 & 349 & 94.07 & 22 & 5.93 \\
\hline \multirow{2}{*}{$\begin{array}{l}\text { Education } \\
\text { (monthly income in Ariary) }\end{array}$} & $80,000-160,000$ & 123 & 24.7 & 99 & 80.49 & 24 & 19.51 \\
\hline & $>160,000$ & 4 & 0.8 & 3 & 74.9 & 1 & 25.1 \\
\hline
\end{tabular}

Comparison of age group, level of schooling, marital status and income level of the 498 interview respondents of Mahabo-Mananivo. 
Table 2 Species known to be medicinal by women in Mahabo-Mananivo

\begin{tabular}{|c|c|c|c|c|c|}
\hline Family & Scientific name & $\begin{array}{l}\text { Vernacular } \\
\text { name }\end{array}$ & Part used & Disease treated & Distribution $[6,25]$ \\
\hline AMARANTHACEAE & Amaranthus sp & Anampatsa & bark & intestinal parasites & \\
\hline AMARANTHACEAE & $\begin{array}{l}\text { Chenopodium } \\
\text { ambrosioides L. }\end{array}$ & Taimboritsiloza & $\begin{array}{l}\text { Entire } \\
\text { plant }\end{array}$ & Placental apposition - Parasites - Nosebleeds & $\begin{array}{l}\text { Naturalized in } \\
\text { Madagascar }\end{array}$ \\
\hline ANACARDIACEAE & Mangifera indica $\mathrm{L}$. & Manga & Bark Root & $\begin{array}{c}\text { Evacuation of the placenta - Diarrhea - Hemorrhoid - } \\
\text { Leucorrhoea - Dental Disease - Gonorrhea }\end{array}$ & $\begin{array}{l}\text { Naturalized in } \\
\text { Madagascar }\end{array}$ \\
\hline ANACARDIACEAE & $\begin{array}{l}\text { Rhus taratana } \\
\text { (Baker) H. Perrier }\end{array}$ & Taranta & Leaf & Poisoning - Convulsions - Epilepsy - Stomach pain & $\begin{array}{l}\text { Endemic to } \\
\text { Madagascar }\end{array}$ \\
\hline ANACARDIACEAE & $\begin{array}{l}\text { Sclerocarya birrea } \\
\text { (A. Rich.) Hochst. }\end{array}$ & Sakoa & Leaf & Venereal diseases - Sedative - Astringent - Spider Bite & Comoros, Africa \\
\hline ANNONACEAE & $\begin{array}{c}\text { Annona reticulata } \\
\mathrm{L} \text {. }\end{array}$ & Coeur de Boeuf & Leaf & Evacuation of the placenta & \\
\hline ANNONACEAE & Annona sp. & Sarisoky & Leaf & Stomach pain & \\
\hline APHLOIACEAE & $\begin{array}{l}\text { Aphloia theiformis } \\
\text { (Vahl) Benn. }\end{array}$ & Fandramana & Leaf, Bark & $\begin{array}{c}\text { Evacuation of the placenta - Malaria - Tuberculosis - } \\
\text { Sore throat - Heartburn }\end{array}$ & $\begin{array}{l}\text { Comoros, } \\
\text { Mascarenes, } \\
\text { Seychelles, Africa }\end{array}$ \\
\hline APOCYNACEAE & $\begin{array}{l}\text { Catharanthus } \\
\text { roseus (L.) G. Don }\end{array}$ & Vonenina & $\begin{array}{l}\text { Entire } \\
\text { plant, Root }\end{array}$ & Stomach pain - Pancreas pain - Cancer & $\begin{array}{l}\text { Endemic to } \\
\text { Madagascar }\end{array}$ \\
\hline APOCYNACEAE & $\begin{array}{c}\text { Petchia } \\
\text { erythrocarpa } \\
\text { (Vatke) Leeuwenb. }\end{array}$ & Hentona & Bark & Malaria & Comoros \\
\hline APOCYNACEAE & $\begin{array}{l}\text { Petchia } \\
\text { madagascariensis } \\
\text { (A. DC.) } \\
\text { Leeuwenb. }\end{array}$ & Kabokala & Leaf & Insect bites & $\begin{array}{l}\text { Endemic to } \\
\text { Madagascar }\end{array}$ \\
\hline APOCYNACEAE & $\begin{array}{l}\text { Voacanga thouarsii } \\
\text { Roem. \& Schult. }\end{array}$ & Kaboky & $\begin{array}{l}\text { Leaf- Latex } \\
\text { - Roots - } \\
\text { Bark-seeds }\end{array}$ & $\begin{array}{c}\text { Evacuation of the placenta - Hypertension - Heart } \\
\text { problems-wounds - Boils - Gonorrhea-Eczema - Sca- } \\
\text { bies - Fungal Infections - Rheumatism - Stomach } \\
\text { pain }\end{array}$ & Africa \\
\hline AQUIFOLIACEAE & Ilex mitis (L.) Radlk. & Hazondrano & Leaf & Bad luck & Africa \\
\hline ARACEAE & $\begin{array}{l}\text { Colocasia esculenta } \\
\text { (L.) Schott }\end{array}$ & Saonjo & Leaf & Evacuation of the placenta & $\begin{array}{l}\text { Naturalized in } \\
\text { Madagascar }\end{array}$ \\
\hline ARACEAE & $\begin{array}{l}\text { Typhonodorum } \\
\text { lindleyanum } \\
\text { Schott }\end{array}$ & Via & Leaf, heart & Evacuation of the placenta - Burn - hip problems & $\begin{array}{l}\text { Comoros, } \\
\text { Mascarenes, Africa }\end{array}$ \\
\hline ARALIACEAE & $\begin{array}{l}\text { Schefflera } \\
\text { longipedicellata } \\
\text { (Lecomte) } \\
\text { Bernardi }\end{array}$ & $\begin{array}{l}\text { Membolo - } \\
\text { vatsila }\end{array}$ & & Epilepsy - Cold - Gonorrhea & $\begin{array}{l}\text { Endemic to } \\
\text { Madagascar }\end{array}$ \\
\hline ARALIACEAE & Schefflera sp. & Memboloha & Leaf & Albumin - Worms - Plague - Evacuation of placenta & \\
\hline ASCLEPIADACEAE & Pentopetia sp & Tandrokosy & Leaf, Stem & Eye disease - Jaundice - Gonorrhea & \\
\hline ASPARAGACEAE & $\begin{array}{l}\text { Dracaena reflexa } \\
\text { Lam. }\end{array}$ & Hasina & Leaf - stem & Evacuation of the placenta - Malaria - Epilepsy & Mascarenes, Africa \\
\hline ASPARAGACEAE & $\begin{array}{l}\text { Dracaena reflexa } \\
\text { var. cernua (Jacq.) } \\
\text { Baker }\end{array}$ & Fananaraha & Leaf - stem & Placental apposition - Thinning & \\
\hline ASPLENIACEAE & Asplenium sp. & Apanga malemy & $\begin{array}{l}\text { Entire } \\
\text { plant }\end{array}$ & gonorrhea & \\
\hline ASTERACEAE & $\begin{array}{l}\text { Acanthospermum } \\
\text { hispidum DC. }\end{array}$ & Bakakely & Leaf & Diarrhea & Africa \\
\hline ASTERACEAE & $\begin{array}{l}\text { Ageratum } \\
\text { conyzoides L. }\end{array}$ & Ananjazavavy & flowers & Stomach pain & $\begin{array}{l}\text { Naturalized in } \\
\text { Madagascar }\end{array}$ \\
\hline ASTERACEAE & Emilia sp. & Kitsitsona & Leaf & Eczema - Ulcer & \\
\hline
\end{tabular}


Table 2 Species known to be medicinal by women in Mahabo-Mananivo (Continued)

\begin{tabular}{|c|c|c|c|c|c|}
\hline ASTERACEAE & Emilia sp. & Tsiotsio & Leaf & Apposition of the placenta & \\
\hline ASTERACEAE & Helichrysum sp. & Aferombohitra & Leaf & Scabies & \\
\hline ASTERACEAE & Mimosa pudica $\mathrm{L}$. & Ramoria & Leaf & Hepatitis - Albumin & $\begin{array}{l}\text { Naturalized in } \\
\text { Madagascar }\end{array}$ \\
\hline ASTERACEAE & $\begin{array}{l}\text { Psiadia altissima } \\
\text { (DC.) Drake }\end{array}$ & Dinga & Leaf & Wounds & $\begin{array}{l}\text { Endemic to } \\
\text { Madagascar }\end{array}$ \\
\hline ASTERACEAE & $\begin{array}{l}\text { Sigesbeckia } \\
\text { orientalis L. }\end{array}$ & Tsindaory & Leaf & Wounds & $\begin{array}{l}\text { Naturalized in } \\
\text { Madagascar }\end{array}$ \\
\hline ASTERACEAE & $\begin{array}{l}\text { Vernonia } \\
\text { appendiculata } \\
\text { Less. }\end{array}$ & Asotry & Leaf & tooth decay & $\begin{array}{l}\text { Endemic to } \\
\text { Madagascar }\end{array}$ \\
\hline ASTERACEAE & $\begin{array}{l}\text { Vernonia exserta } \\
\text { Baker }\end{array}$ & Seva & Leaf & Chickenpox - Parasites & $\begin{array}{l}\text { Endemic to } \\
\text { Madagascar }\end{array}$ \\
\hline ASTERACEAE & $\begin{array}{l}\text { Vernoniopsis } \\
\text { caudata (Drake) } \\
\text { Humbert }\end{array}$ & $\begin{array}{l}\text { Maranitry } \\
\text { atoraky }\end{array}$ & Bark & Chickenpox & $\begin{array}{l}\text { Endemic to } \\
\text { Madagascar }\end{array}$ \\
\hline ASTEROPEIACEAE & $\begin{array}{l}\text { Asteropeia } \\
\text { micraster Hallier } \mathrm{f} .\end{array}$ & Manoky mena & Bark, Leaf & $\begin{array}{c}\text { Evacuation of the placenta - Diarrhea - Fatigue - } \\
\text { Mumps }\end{array}$ & $\begin{array}{l}\text { Endemic to } \\
\text { Madagascar }\end{array}$ \\
\hline ASTEROPEIACEAE & $\begin{array}{l}\text { Asteropeia } \\
\text { multiflora Thouars }\end{array}$ & Manoky fotsy & Leaf Bark & $\begin{array}{c}\text { Evacuation of the placenta - Malaria - Parasites - Den- } \\
\text { tal Disease - Gonorrhea Fatigue }\end{array}$ & $\begin{array}{l}\text { Endemic to } \\
\text { Madagascar }\end{array}$ \\
\hline BIGNONIACEAE & $\begin{array}{l}\text { Phyllarthron } \\
\text { madagascariense } \\
\text { K. Schum. }\end{array}$ & Resiriky/ Zahana & Leaf & Malaria - Breastfeeding-Cough - disease of the hip & $\begin{array}{l}\text { Endemic to } \\
\text { Madagascar }\end{array}$ \\
\hline BROMELIACEAE & $\begin{array}{l}\text { Ananas comosus } \\
\text { (L.) Merr. }\end{array}$ & Mananasy & & Intestinal parasites - diarrhea & Tropics \\
\hline BURSERACEAE & Protium sp & Ambihitry & Bark & Abscess - poisoning & \\
\hline CALOHPYLLACEAE & $\begin{array}{l}\text { Calophyllum } \\
\text { inophyllum L. }\end{array}$ & Vintanina & & trigeminal neuralgia & $\begin{array}{l}\text { Comoros, Africa, } \\
\text { New World }\end{array}$ \\
\hline CANELLACEAE & $\begin{array}{l}\text { Cinnamosma } \\
\text { fragrans Baill. }\end{array}$ & Kanely & Bark & $\begin{array}{c}\text { Cold - intestinal parasite - Headaches - Against } \\
\text { poison }\end{array}$ & $\begin{array}{l}\text { Endemic to } \\
\text { Madagascar }\end{array}$ \\
\hline CANELLACEAE & $\begin{array}{l}\text { Cinnamosma } \\
\text { madagascariensis } \\
\text { Danguy }\end{array}$ & Fotsinana & Bark, leaf & $\begin{array}{l}\text { Evacuation of the placenta - Malaria - Hepatitis - Epi- } \\
\text { lepsy - Intoxication - Dysentery - Carrie dental }\end{array}$ & $\begin{array}{l}\text { Endemic to } \\
\text { Madagascar }\end{array}$ \\
\hline CANNABACEAE & Cannabis sativa $\mathrm{L}$. & Rongony & Leaf & Liver disease & \\
\hline CARICACEAE & Carica papaya $\mathrm{L}$. & Paza & $\begin{array}{l}\text { Leaf, Fruit, } \\
\text { seeds, } \\
\text { roots }\end{array}$ & $\begin{array}{c}\text { Breastfeeding - Headaches - Wounds - Menstrual Pain } \\
\text { - Stomach: Ulcer Constipation - Indigestion - Boil - } \\
\text { Cysticercosis - Toxoplasmosis - Cough - Yellow Fever } \\
\text { - Tooth Decay }\end{array}$ & Tropics \\
\hline COMBRETACEAE & $\begin{array}{l}\text { Terminalia catappa } \\
\text { L. }\end{array}$ & Atafa & Leaf & $\begin{array}{c}\text { Ovarian cycle disruption - Albumin - Tension - Stom- } \\
\text { ach pain }\end{array}$ & $\begin{array}{l}\text { Madagascar, } \\
\text { Comoros, } \\
\text { Seychelles, Africa }\end{array}$ \\
\hline CONNARACEAE & $\begin{array}{l}\text { Agelaea pentagyna } \\
\text { (Lam.) Baill. }\end{array}$ & Rangahtsara & Bark & Gonorrhea - Aphrodisiac - Stomach ache & $\begin{array}{c}\text { Comoros, } \\
\text { Mascarenes, Africa }\end{array}$ \\
\hline CONVOLVULACEAE & $\begin{array}{l}\text { Ipomoea batatas } \\
\text { (L.) Lam. }\end{array}$ & Vomanga & Leaf & Pregnancy - Evacuation of the placenta - Insect stings & $\begin{array}{l}\text { Naturalized in } \\
\text { Madagascar }\end{array}$ \\
\hline CRASSULACEAE & $\begin{array}{l}\text { Kalanchoe prolifera } \\
\text { (Bowie ex Hook.) } \\
\text { Hamet }\end{array}$ & Silafafa & Leaf & Asthma - Cough - Rheumatism & $\begin{array}{l}\text { Endemic to } \\
\text { Madagascar }\end{array}$ \\
\hline CUCURBITACEAE & $\begin{array}{l}\text { Cucurbita maxima } \\
\text { Duchesne }\end{array}$ & Voatavo & Leaf & Fever - colic & $\begin{array}{l}\text { Naturalized in } \\
\text { Madagascar }\end{array}$ \\
\hline CYPERACEAE & $\begin{array}{l}\text { Cyperus papyrus } \\
\text { subsp. } \\
\text { madagascariensis } \\
\text { (Willd.) Kük. }\end{array}$ & Zozoro & & Difficulty after childbirth - painful spasms & \\
\hline CYPERACEAE & & Ahibita & & Evacuation of placenta-Malaria - Tuberculosis & \\
\hline
\end{tabular}


Table 2 Species known to be medicinal by women in Mahabo-Mananivo (Continued)

\begin{tabular}{|c|c|c|c|c|c|}
\hline & $\begin{array}{l}\text { Pycreus mundtii } \\
\text { Cherm. }\end{array}$ & & $\begin{array}{l}\text { Entire } \\
\text { plant }\end{array}$ & & $\begin{array}{l}\text { Mascarenes, Africa, } \\
\text { New World }\end{array}$ \\
\hline EBENACEAE & Diospyros sp & Hazominty & Leaf & Malaria & \\
\hline ERICACEAE & $\begin{array}{l}\text { Agauria salicifolia } \\
\text { (Comm. ex Lam.) } \\
\text { Hook. f. ex Oliv. }\end{array}$ & Haronga-panihy & Leaf & Scabies (Adult) - Wounds - Ulcers & Mascarenes, Africa \\
\hline ERICACEAE & Erica sp. & Anjavidy & $\begin{array}{l}\text { Leaf Stem } \\
\text { leaves }\end{array}$ & Evacuation of placenta-Pneumonia - Syphilis & \\
\hline ERYTHROXYLACEAE & $\begin{array}{l}\text { Erythroxylum } \\
\text { ferrugineum Cav. }\end{array}$ & Menahihy & Bark Leaf & Evacuation of the placenta - Diarrhea - Anemia & $\begin{array}{l}\text { Endemic to } \\
\text { Madagascar }\end{array}$ \\
\hline ERYTHROXYLACEAE & $\begin{array}{l}\text { Erythroxylum } \\
\text { gerrardii Baker }\end{array}$ & Fanjoana & Leaf Bark & Yellow fever - Epilepsy & Africa \\
\hline EUPHORBIACEAE & $\begin{array}{l}\text { Croton noronhae } \\
\text { Baill. }\end{array}$ & Tsiavadika & Bark-Leaf & Placental apposition - Malaria - Cough & $\begin{array}{l}\text { Endemic to } \\
\text { Madagascar }\end{array}$ \\
\hline EUPHORBIACEAE & Euphorbia hirta L. & Jean Robert & $\begin{array}{l}\text { Entire } \\
\text { plant }\end{array}$ & Gonorrhea - Dysentery - Albumin & $\begin{array}{l}\text { Naturalized in } \\
\text { Madagascar }\end{array}$ \\
\hline EUPHORBIACEAE & Jatropha curcas L. & Savoa & Leaf Latex & $\begin{array}{l}\text { Evacuation of placenta Asthma - Dental Disease - } \\
\qquad \text { Pneumonia }\end{array}$ & $\begin{array}{l}\text { Naturalized in } \\
\text { Madagascar }\end{array}$ \\
\hline EUPHORBIACEAE & $\begin{array}{c}\text { Macaranga } \\
\text { oblongifolia Baill. }\end{array}$ & Mokarana & Leaf & Malaria - Diarrhea & $\begin{array}{l}\text { Endemic to } \\
\text { Madagascar }\end{array}$ \\
\hline EUPHORBIACEAE & Macaranga sp & Mokarana & Leaf & Diarrhea & \\
\hline EUPHORBIACEAE & $\begin{array}{l}\text { Manihot utilissima } \\
\text { Pohl }\end{array}$ & Kazaha & Leaf & Gonorrhea - painful spasms - Pneumonia - Boil & \\
\hline EUPHORBIACEAE & $\begin{array}{c}\text { Suregada } \\
\text { boiviniana Baill. }\end{array}$ & Lelangana & Leaf & Placental apposition - Dysentery - Epilepsy-Malaria & $\begin{array}{l}\text { Endemic to } \\
\text { Madagascar }\end{array}$ \\
\hline FABACEAE & $\begin{array}{c}\text { Albizia gummifera } \\
\text { (J.F. Gmel.) C.A. } \\
\text { Sm. }\end{array}$ & Volomborona & Leaf & Fatigue - Cough & Africa \\
\hline FABACEAE & $\begin{array}{l}\text { Cajanus cajan (L.) } \\
\text { Huth }\end{array}$ & Ambatry & Leaf & Evacuation of the placenta - Tension & $\begin{array}{l}\text { Naturalized in } \\
\text { Madagascar }\end{array}$ \\
\hline FABACEAE & $\begin{array}{l}\text { Chamaecrista } \\
\text { mimosoides (L.) } \\
\text { Greene }\end{array}$ & Quatre épingles & Leaf & Thrush - Schistosomiasis & \\
\hline FABACEAE & $\begin{array}{l}\text { Intsia bijuga } \\
\text { (Colebr.) Kuntze }\end{array}$ & Hintsy & Leaf & Placental apposition - Cough & Mascarenes, Africa \\
\hline FABACEAE & Mimosa pudica L. & Ramoria & & Pelvic pain - Nervousness - Diuretic & $\begin{array}{l}\text { Naturalized in } \\
\text { Madagascar }\end{array}$ \\
\hline FABACEAE & $\begin{array}{l}\text { Senna alata (L.) } \\
\quad \text { Roxb. }\end{array}$ & Quatre épingles & Leaf & Hypertension & $\begin{array}{l}\text { Naturalized in } \\
\text { Madagascar }\end{array}$ \\
\hline GENTIANACEAE & $\begin{array}{c}\text { Tachiadenus } \\
\text { carinatus (Desr.) } \\
\text { Griseb. }\end{array}$ & Malanilava & $\begin{array}{l}\text { Entire } \\
\text { plant }\end{array}$ & Diarrhea & $\begin{array}{l}\text { Endemic to } \\
\text { Madagascar }\end{array}$ \\
\hline GLEICHENIACEAE & $\begin{array}{l}\text { Sticherus flagellaris } \\
\text { (Bory ex Willd.) } \\
\quad \text { Ching }\end{array}$ & Ringotra & Leaf & Diarrhea - Measles - Vomiting - Coughing & $\begin{array}{l}\text { Mascarenes, } \\
\text { Comoros }\end{array}$ \\
\hline HYPERICACEAE & $\begin{array}{c}\text { Harungana } \\
\text { madagascariensis } \\
\text { Lam. ex Poir. }\end{array}$ & Harongana & Bud Leaf & $\begin{array}{c}\text { Gonorrhea - heart disease - Albumin - Asthma - Boil- } \\
\text { Diarrhea }\end{array}$ & $\begin{array}{l}\text { Comoros, } \\
\text { Mascarenes, Africa }\end{array}$ \\
\hline ICACINACEAE & $\begin{array}{l}\text { Cassinopsis } \\
\text { madagascariensis } \\
\text { Baill. }\end{array}$ & Valotry & Leaf - Bark & Cough - Itching - Syphilis & $\begin{array}{l}\text { Endemic to } \\
\text { Madagascar }\end{array}$ \\
\hline LAMIACEAE & $\begin{array}{l}\text { Ocimum } \\
\text { gratissimum L. }\end{array}$ & Romba be & Leaf & $\begin{array}{c}\text { Placental apposition - Asthma - Albumin - Headache } \\
\text { - Dental Disease }\end{array}$ & $\begin{array}{c}\text { Comoros, } \\
\text { Mascarenes, } \\
\text { Seychelles, Africa, } \\
\text { Asia }\end{array}$ \\
\hline
\end{tabular}


Table 2 Species known to be medicinal by women in Mahabo-Mananivo (Continued)

\begin{tabular}{|c|c|c|c|c|c|}
\hline LAMIACEAE & $\begin{array}{l}\text { Salvia coccinea } \\
\text { Buc'hoz ex Etl. }\end{array}$ & $\begin{array}{l}\text { Romba } \\
\text { madinika }\end{array}$ & Leaf & parasites & $\begin{array}{l}\text { Naturalized in } \\
\text { Madagascar }\end{array}$ \\
\hline LAURACEAE & $\begin{array}{l}\text { Persea americana } \\
\text { Mill. }\end{array}$ & Zavoka & Leaf & Diarrhea - Apposition of placental - Cough & $\begin{array}{l}\text { Naturalized in } \\
\text { Madagascar }\end{array}$ \\
\hline LECYTHIDACEAE & $\begin{array}{c}\text { Barringtonia } \\
\text { racemosa }(\mathrm{L} .) \\
\text { Spreng. }\end{array}$ & Fotatry & Leaf & Placental apposition - Scabies - Tetanus & $\begin{array}{c}\text { Comoros, } \\
\text { Australiasia, Africa }\end{array}$ \\
\hline LILIACEAE & $\begin{array}{l}\text { Asparagus } \\
\text { simulans Baker }\end{array}$ & Ahitsifantatry & $\begin{array}{l}\text { Entire } \\
\text { plant }\end{array}$ & Epilepsy - Stomach pain & $\begin{array}{l}\text { Endemic to } \\
\text { Madagascar }\end{array}$ \\
\hline LOMARIOPSIDACEAE & $\begin{array}{c}\text { Nephrolepis } \\
\text { cordifolia (L.) C. } \\
\text { Presl }\end{array}$ & Mitsisiloha & $\begin{array}{l}\text { Entire } \\
\text { plant }\end{array}$ & Malaria & $\begin{array}{c}\text { Mascarenes, } \\
\text { Australasia, } \\
\text { Seychelle, Afria, } \\
\text { Asia, New World }\end{array}$ \\
\hline LORANTHACEAE & Bakerella sp & Velomiato & $\begin{array}{l}\text { Entire } \\
\text { plant }\end{array}$ & Convulsion - Cough - Boil & \\
\hline LYCOPODIACEAE & $\begin{array}{l}\text { Lycopodiella } \\
\text { cernua (L.) Pic. } \\
\text { Serm. }\end{array}$ & Tongotsokina & $\begin{array}{l}\text { Entire } \\
\text { plant }\end{array}$ & $\begin{array}{c}\text { Asthma - Epilepsy - Pelvic Pain - Gonorrhea - Cough - } \\
\text { Hypertension }\end{array}$ & $\begin{array}{l}\text { Mascarenes, Africa, } \\
\text { Asia, New World }\end{array}$ \\
\hline LYCOPODIACEAE & $\begin{array}{l}\text { Lycopodium } \\
\text { clavatum L. }\end{array}$ & Dito & Leaf & $\begin{array}{c}\text { Pregnant - Placental apposition - Gonorrhea - Filaria- } \\
\text { sis - Malaria }\end{array}$ & $\begin{array}{l}\text { Comoros, } \\
\text { Mascarenes, Africa }\end{array}$ \\
\hline MELASTOMACEAE & $\begin{array}{c}\text { Clidemia hirta (L.) } \\
\text { D. Don }\end{array}$ & Voatrotrokala & Leaf & Wounds & $\begin{array}{c}\text { Naturalized in } \\
\text { Madagascar }\end{array}$ \\
\hline MELASTOMACEAE & Dichaetanthera sp & Felabarika & Leaf & diarrhea & \\
\hline MELASTOMACEAE & Medinilla & Takasina & & Cough & \\
\hline MELIACEAE & Melia azedarach L. & Voandelaka & Leaf & Fatigue & $\begin{array}{l}\text { Naturalized in } \\
\text { Madagascar }\end{array}$ \\
\hline MENIPERMACEAE & $\begin{array}{l}\text { Burasaia australis } \\
\text { Scott-Elliot }\end{array}$ & Sompatry & Leaf & $\begin{array}{c}\text { Intoxication - Convulsion - Dental Disease - Malaria - } \\
\text { Medicinal plant magic }\end{array}$ & $\begin{array}{l}\text { Endemic to } \\
\text { Madagascar }\end{array}$ \\
\hline MOLLUGINACEAE & $\begin{array}{l}\text { Mollugo nudicaulis } \\
\text { Lam. }\end{array}$ & Aferotany & $\begin{array}{l}\text { Entire } \\
\text { plant }\end{array}$ & $\begin{array}{c}\text { Malaria - Albumin - Convulsion - Cough - Diarrhea - } \\
\text { Diarrhea - Blood loss - Scabies }\end{array}$ & $\begin{array}{l}\text { Australasia, Africa, } \\
\text { New World }\end{array}$ \\
\hline MONIMIACEAE & $\begin{array}{l}\text { Tambourissa castri- } \\
\text { delphinii Cavaco }\end{array}$ & Amborabe & Leaf & Placental apposition - Dysentery & $\begin{array}{l}\text { Endemic to } \\
\text { Madagascar }\end{array}$ \\
\hline MONIMIACEAE & $\begin{array}{c}\text { Tambourissa } \\
\text { parvifolia Baker }\end{array}$ & Ambora & Leaf & Filariasis - Loss of blood & $\begin{array}{l}\text { Endemic to } \\
\text { Madagascar }\end{array}$ \\
\hline MORACEAE & $\begin{array}{l}\text { Artocarpus altilis } \\
\text { (Parkinson) } \\
\text { Fosberg }\end{array}$ & Soanambo & Leaf & Diarrhea & \\
\hline MORACEAE & $\begin{array}{l}\text { Ficus polita subsp. } \\
\text { polita }\end{array}$ & Mandresy & Leaf & Placental apposition - bilious - Gonorrhea - Syphilis & \\
\hline MORACEAE & $\begin{array}{l}\text { Ficus reflexa } \\
\text { Thunb. }\end{array}$ & Laza & Leaf & Pelvic pain - Gonorrhea & $\begin{array}{l}\text { Comoros, } \\
\text { Mascarenes, } \\
\text { Seychelles }\end{array}$ \\
\hline MUSACEAE & $\begin{array}{c}\text { Musa } \times \text { paradisiaca } \\
\text { L. }\end{array}$ & Akondro & Leaf - fruit & $\begin{array}{c}\text { Placental apposition - Diabetes - Prevents tooth } \\
\text { decay - Diarrhea - Wounds }\end{array}$ & $\begin{array}{l}\text { Naturalized in } \\
\text { Madagascar }\end{array}$ \\
\hline MYRICACEAE & $\begin{array}{c}\text { Morella spathulata } \\
\text { (Mirb.) Verdc. \& } \\
\text { Polhill }\end{array}$ & Hazosiay & Leaf & $\begin{array}{c}\text { Placental apposition - Malaria - Cough - Stomach } \\
\text { Pain - Dental Disease- Injury }\end{array}$ & Africa \\
\hline MYRISTICACEAE & $\begin{array}{c}\text { Brochoneura } \\
\text { acuminata (Lam.) } \\
\text { Warb. }\end{array}$ & Raraha & Leaf & Injury - Scabies - Abscess & $\begin{array}{l}\text { Endemic to } \\
\text { Madagascar }\end{array}$ \\
\hline MYRTACEAE & Melaleuca & Kininy bonaky & Leaf & Placental apposition - Cold & $\begin{array}{l}\text { Naturalized in } \\
\text { Madagascar }\end{array}$ \\
\hline MYRTACEAE & $\begin{array}{l}\text { Psidium } \\
\text { cattleyanum } \\
\text { Sabine }\end{array}$ & Angavombazaha & Leaf & Diarrhea & $\begin{array}{l}\text { Naturalized in } \\
\text { Madagascar }\end{array}$ \\
\hline
\end{tabular}


Table 2 Species known to be medicinal by women in Mahabo-Mananivo (Continued)

\begin{tabular}{|c|c|c|c|c|c|}
\hline MYRTACEAE & Psidium guajava $\mathrm{L}$. & Angavogasy & Leaf & Malaria - Colic stomach - diarrhea - dysentery & $\begin{array}{l}\text { Naturalized in } \\
\text { Madagascar }\end{array}$ \\
\hline MYRTACEAE & Psidium guajava $\mathrm{L}$. & Angavofotsy & $\begin{array}{l}\text { Leafs } \\
\text { Roots }\end{array}$ & Diarrhea - Vomiting Boil & $\begin{array}{l}\text { Naturalized in } \\
\text { Madagascar }\end{array}$ \\
\hline MYRTACEAE & $\begin{array}{l}\text { Syzygium } \\
\text { aromaticum (L.) } \\
\text { Merr. \& L.M. Perry }\end{array}$ & Jirofo & Leaf & Placental apposition - Dental Disease - Malaria & \\
\hline MYRTACEAE & $\begin{array}{l}\text { Syzygium bernieri } \\
\text { (Drake) Labat \& G. } \\
\text { E. Schatz }\end{array}$ & Rotry & Bark-Leaf & $\begin{array}{l}\text { Placental apposition - diarrhea - Dentistry Diseases - } \\
\text { Scabies }\end{array}$ & $\begin{array}{l}\text { Endemic to } \\
\text { Madagascar }\end{array}$ \\
\hline MYRTACEAE & $\begin{array}{l}\text { Syzygium } \\
\text { emirnense (Baker) } \\
\text { Labat \& G.E. } \\
\text { Schatz }\end{array}$ & Rotry & Bark-Leaf & Placental apposition - Dentistry Diseases - Scabies & Mascarenes \\
\hline NEPENTHACEAE & $\begin{array}{l}\text { Nepenthes } \\
\text { madagascariensis } \\
\text { Poir. }\end{array}$ & Kapilanomba & $\begin{array}{l}\text { Entire } \\
\text { plantEau } \\
\text { dans l'urne }\end{array}$ & $\begin{array}{c}\text { Adhesion of placental-malaria-Albumin - Filariasis - } \\
\text { Gonorrhea Syphilis-ear disease }\end{array}$ & $\begin{array}{l}\text { Endemic to } \\
\text { Madagascar }\end{array}$ \\
\hline NYMPHEACEAE & $\begin{array}{c}\text { Nymphaea } \\
\text { nouchali Burm. f. }\end{array}$ & Tatamo & Tubers & Hemorrhoids - Pelvic Pain & $\begin{array}{l}\text { Comoros, } \\
\text { Mascarenes, Africa, } \\
\text { Asia }\end{array}$ \\
\hline OLACACEAE & $\begin{array}{l}\text { Olax emirnensis } \\
\text { Baker }\end{array}$ & Soazanahary & Leaf & $\begin{array}{c}\text { Placental apposition - Malaria - Hepatitis - Epilepsy - } \\
\text { Self-defense against witchcraft - Dysentery - Fatigue - } \\
\text { Medicinal plant magic }\end{array}$ & $\begin{array}{l}\text { Endemic to } \\
\text { Madagascar }\end{array}$ \\
\hline OPHIOGLOSSACEAE & Ophioglossum L. & Tsipanga & Leaf & Childbirth & \\
\hline ORCHIDACEAE & Angraecum sp. & Valily & $\begin{array}{l}\text { Entire } \\
\text { plant }\end{array}$ & fortifying & \\
\hline PANDANACEAE & Pandanus sp & Vakoana & Leaf & Fatigue - Impotence & \\
\hline PASSIFLORACEAE & $\begin{array}{l}\text { Passiflora edulis } \\
\text { Sims }\end{array}$ & Garana & Leaf & Tension - Parasites & $\begin{array}{l}\text { naturalized in } \\
\text { Madagascar }\end{array}$ \\
\hline PHYLLANTHACEAE & Phyllanthus sp & Masombero & Leaf & Apposition of the placenta & \\
\hline PHYSENACEAE & $\begin{array}{l}\text { Physena } \\
\text { madagascariensis } \\
\text { Thouars ex Tul. }\end{array}$ & Resojo & Bark & Sore throat - Anemia - Against poison & $\begin{array}{l}\text { Endemic to } \\
\text { Madagascar }\end{array}$ \\
\hline PIPERACEAE & Piper nigrum L. & Poivre & Seeds & Dental disease - Poultice - Joint pain & \\
\hline PITTOSPORACEAE & $\begin{array}{l}\text { Pittosporum } \\
\text { verticillatum Bojer }\end{array}$ & Memboloha & Leaf- Bark & Malaria - Adhesion of placental & $\begin{array}{l}\text { Endemic to } \\
\text { Madagascar }\end{array}$ \\
\hline POACEAE & $\begin{array}{l}\text { Cymbopogon } \\
\text { citratus (DC.) Stapf }\end{array}$ & Veromanitra & $\begin{array}{l}\text { Entire } \\
\text { plant }\end{array}$ & Fever & $\begin{array}{l}\text { Australasia/Pacific, } \\
\text { Africa, Asia, New } \\
\text { World }\end{array}$ \\
\hline POACEAE & $\begin{array}{l}\text { Cynodon dactylon } \\
\text { (L.) Pers. }\end{array}$ & Kindresy & $\begin{array}{l}\text { Entire } \\
\text { plant }\end{array}$ & $\begin{array}{l}\text { Albumin - Malaria - Liver Disease - Menstrual Pain - } \\
\text { Laxative }\end{array}$ & $\begin{array}{l}\text { Australasia, Africa, } \\
\text { New World }\end{array}$ \\
\hline POACEAE & $\begin{array}{l}\text { Eleusine indica (L.) } \\
\text { Gaertn. }\end{array}$ & Tsipihipihina & $\begin{array}{l}\text { Entire } \\
\text { plant }\end{array}$ & Stomach pain & $\begin{array}{l}\text { Africa, Asia, New } \\
\text { World }\end{array}$ \\
\hline POACEAE & $\begin{array}{l}\text { Hyparrhenia rufa } \\
\text { (Nees) Stapf }\end{array}$ & Verofehana & $\begin{array}{l}\text { Entire } \\
\text { plant }\end{array}$ & Epilepsy - Cracks skin of the feet & $\begin{array}{l}\text { Africa, Asia, New } \\
\text { World }\end{array}$ \\
\hline POACEAE & $\begin{array}{l}\text { Imperata cylindrica } \\
\text { (L.) Raeusch. }\end{array}$ & Tenina & Leaf & $\begin{array}{c}\text { Intoxication - Gonorrhea - Pneumonia - Tonsillitis - } \\
\text { Measles - Tension }\end{array}$ & $\begin{array}{l}\text { Naturalized in } \\
\text { Madagascar }\end{array}$ \\
\hline POACEAE & Oryza sativa $L$. & Vary & Bud & birth & $\begin{array}{l}\text { cultivated in } \\
\text { Madagascar }\end{array}$ \\
\hline POACEAE & $\begin{array}{c}\text { Panicum } \\
\text { maximum Jacq. }\end{array}$ & Ahitry & Leafs & Wounds & $\begin{array}{c}\text { Madagascar, Africa, } \\
\text { New World }\end{array}$ \\
\hline POACEAE & $\begin{array}{l}\text { Sporobolus } \\
\text { africanus (Poir.) } \\
\text { Robyns \& Tournay }\end{array}$ & Ahitry & $\begin{array}{l}\text { Entire } \\
\text { plant }\end{array}$ & Allergy & $\begin{array}{c}\text { Australasia, Africa, } \\
\text { New World }\end{array}$ \\
\hline POACEAE & Zea mays $\mathrm{L}$. & Katsaka & Barbe & gonorrhea & \\
\hline
\end{tabular}


Table 2 Species known to be medicinal by women in Mahabo-Mananivo (Continued)

\begin{tabular}{|c|c|c|c|c|c|}
\hline & & & & & $\begin{array}{l}\text { cultivated in } \\
\text { Madagascar }\end{array}$ \\
\hline POLYGONACEAE & $\begin{array}{c}\text { Persicaria } \\
\text { senegalensis } \\
\text { (Meisn.) Soják }\end{array}$ & Fotsimbarinako & Root & Malaria & $\begin{array}{l}\text { Naturalized in } \\
\text { Madagascar }\end{array}$ \\
\hline RUBIACEAE & Canthium sp. & Fotsikahitry & Leaf & Epilepsy & \\
\hline RUBIACEAE & Coffea sp. & Kafe & Leaf & Malaria & \\
\hline RUBIACEAE & $\begin{array}{l}\text { Danais cernua } \\
\text { Baker }\end{array}$ & Fangalalemy & Leaf Bark & Syphilis - Tooth Decay & $\begin{array}{l}\text { Endemic to } \\
\text { Madagascar }\end{array}$ \\
\hline RUBIACEAE & Paederia foetida $\mathrm{L}$. & Ahimembo & Leaf & Evacuation of the placenta - Headaches & \\
\hline RUBIACEAE & Psychotria sp & Sariloa & Leaf & diarrhea & \\
\hline RUTACEAE & $\begin{array}{l}\text { Cedrelopsis grevei } \\
\text { Baill. }\end{array}$ & Hafatraina & Leaf Bark & Stomachaches - Acne & $\begin{array}{l}\text { Endemic to } \\
\text { Madagascar }\end{array}$ \\
\hline RUTACEAE & Citrus aurantium L. & $\begin{array}{l}\text { Voasary } \\
\text { makirana }\end{array}$ & Fruit & Cough - Malaria & $\begin{array}{l}\text { Naturalized in } \\
\text { Madagascar }\end{array}$ \\
\hline RUTACEAE & $\begin{array}{l}\text { Citrus sinensis (L.) } \\
\text { Osbeck }\end{array}$ & Voangy gasy & Leaf & Evacuation of placenta-Malaria & \\
\hline SALICACEAE & $\begin{array}{l}\text { Homalium axillare } \\
\text { (Lam.) Benth. }\end{array}$ & Fotsiakara & Bark & burns & $\begin{array}{l}\text { Endemic to } \\
\text { Madagascar }\end{array}$ \\
\hline SALICACEAE & Scolopia sp & Hazofotsy & Bark & Rheumatism & \\
\hline SAPINDACEAE & $\begin{array}{l}\text { Litchi chinensis } \\
\text { Sonn. }\end{array}$ & Letchis & Leaf & diarrhea & $\begin{array}{l}\text { cultivated in } \\
\text { Madagascar }\end{array}$ \\
\hline SARCOLAENACEAE & $\begin{array}{l}\text { Leptolaena } \\
\text { pauciflora Baker }\end{array}$ & Fatra & Bark & Syphilis & $\begin{array}{l}\text { Endemic to } \\
\text { Madagascar }\end{array}$ \\
\hline SARCOLAENACEAE & $\begin{array}{l}\text { Sarcolaena } \\
\text { multiflora Thouars }\end{array}$ & Hela & Leaf & Evacuation of placenta & $\begin{array}{l}\text { Endemic to } \\
\text { Madagascar }\end{array}$ \\
\hline SCHIZACACEAE & $\begin{array}{l}\text { Lygodium } \\
\text { lanceolatum Desv. }\end{array}$ & Sofin'akanga & Leaf & $\begin{array}{c}\text { Pancrea pain - Gonorrhea - Tension - Evacuation of } \\
\text { placenta }\end{array}$ & $\begin{array}{l}\text { Native to } \\
\text { Madagascar }\end{array}$ \\
\hline SIMARUBACEAE & Quassia sp. & Rembiky & Leaf & Aphrodisiac & \\
\hline SMILACEAE & $\begin{array}{l}\text { Smilax anceps } \\
\quad \text { Willd. }\end{array}$ & Roindambo & Leaf & Convulsion - Pregnancy - Fatigue - Boil & $\begin{array}{c}\text { Comoros, } \\
\text { Mascarenes, Africa }\end{array}$ \\
\hline SOLANACEAE & $\begin{array}{c}\text { Capsicum annuum } \\
\mathrm{L} .\end{array}$ & Sakaipilo & Fruit & Rheumatism - Pain & $\begin{array}{l}\text { Naturalized in } \\
\text { Madagascar }\end{array}$ \\
\hline SOLANACEAE & Datura inoxia Mill. & Ramiary & Leaf & Asthma - Calming & $\begin{array}{l}\text { Naturalized in } \\
\text { Madagascar }\end{array}$ \\
\hline SOLANACEAE & $\begin{array}{l}\text { Nicotiana tabacum } \\
\mathrm{L} \text {. }\end{array}$ & Paraky & Leaf & Nosebleed & $\begin{array}{l}\text { Naturalized in } \\
\text { Madagascar }\end{array}$ \\
\hline SOLANACEAE & $\begin{array}{c}\text { Solanum } \\
\text { erythracanthum } \\
\text { Bojer ex Dunal }\end{array}$ & Angivy & Fruit & Cough & $\begin{array}{l}\text { Endemic to } \\
\text { Madagascar }\end{array}$ \\
\hline STILBACEAE & $\begin{array}{l}\text { Nuxia capitata } \\
\text { Baker }\end{array}$ & Valanirana & Leaf & Cough - Tonic - Tapeworm & $\begin{array}{l}\text { Endemic to } \\
\text { Madagascar }\end{array}$ \\
\hline STRELITZIACEAE & $\begin{array}{c}\text { Ravenala } \\
\text { madagascariensis } \\
\text { Sonn. }\end{array}$ & Ravinala & Leaf & Tension & $\begin{array}{l}\text { Endemic to } \\
\text { Madagascar }\end{array}$ \\
\hline TACCACEAE & $\begin{array}{c}\text { Tacca } \\
\text { leontopetaloides } \\
\text { (L.) Kuntze }\end{array}$ & Tavolo & Tuber & Malnutrition & $\begin{array}{l}\text { Naturalized in } \\
\text { Madagascar }\end{array}$ \\
\hline THYMELACEAE & $\begin{array}{c}\text { Gnidia danguyana } \\
\text { Leandri }\end{array}$ & Avoha & Leaf & Bleeding - Parasites & $\begin{array}{l}\text { Endemic to } \\
\text { Madagascar }\end{array}$ \\
\hline ULMACEAE & $\begin{array}{l}\text { Trema orientalis } \\
\text { (L.) Blume }\end{array}$ & $\begin{array}{c}\text { Andrarezina / } \\
\text { Vakoky }\end{array}$ & Leaf & Evacuation of the placenta - Dental Disease & Africa \\
\hline VACCINACEAE & Vaccinium sp. & Voakaramy & Leaf & Anemia - Diabetes & \\
\hline
\end{tabular}


Table 2 Species known to be medicinal by women in Mahabo-Mananivo (Continued)

\begin{tabular}{|c|c|c|c|c|c|}
\hline ZINGIBERACEAE & $\begin{array}{c}\text { Aframomum } \\
\text { angustifolium } \\
\text { (Sonn.) K. Schum. }\end{array}$ & Longoza & Leaf & Splinter & Africa \\
\hline ZINGIBERACEAE & Curcuma longa L. & Tamotamo & Leaf & Albumin - Pregnancy - Malaria - Jaundice Viral & \\
\hline ZINGIBERACEAE & $\begin{array}{l}\text { Hedychium } \\
\text { coronarium J. } \\
\text { Koenig }\end{array}$ & Longoza & Leaf & Evacuation of the placenta - Scabies & \\
\hline ZINGIBERACEAE & $\begin{array}{l}\text { Zingiber officinale } \\
\text { Roscoe }\end{array}$ & Sakaintany & $\begin{array}{l}\text { Tuber - } \\
\text { Leaf }\end{array}$ & $\begin{array}{c}\text { Pregnancy: Nausea - Evacuation of placenta-cough- } \\
\text { diarrhea }\end{array}$ & \\
\hline
\end{tabular}

Complete list of the vernacular names, scientific identification, use and distribution of all the species mentioned during ethnobotanical interviews.

respondents are single mothers who prefer to practice traditional care during childbirth and/or childhood diseases.

\section{Diversity of medicinal plants and their application}

152 medicinal plants were recorded during our ethnobotanical interviews as part of the collective women's pharmacopeia. The diversity of medicinal plants in the botanical groups shows that dicotyledons have a very high percentage of use $(87 \%)$, followed by $8 \%$ of monocotyledons and finally $5 \%$ of pteridophytes. The most important medicinal families are: Asteraceae (11 species), Poaceae and (9 species), Myrtaceae, Euphorbiaceae and Fabaceae (6 species each), Rubiaceae (5 species), Apocynaceae and Zingiberaceae (4 species each), Anacardiaceae, Moraceae, Melastomataceae and Solanaceae (3 species each). Our findings illustrate the most well known and cited species by women have a high rate of endemism or regional nativity. (Table 2).

Medicinal plants are mainly used in the care of the digestive system (53.95\%), followed by reproductive system (49.34\%), then the circulatory system with $42.76 \%$. Then, the plants used against skin diseases have a frequency of use of $28.29 \%$, those used against diseases of the respiratory system with $20.39 \%$. Eighteen percent (18\%) of plants are taken for the care of diseases related to nervous systems, those used against diseases associated with hearing and visual are a minority ( $0.66 \%$ only) (Figure 2$)$.

In the rural commune of Mahabo-Mananivo, leaves are most often cited as the part used for medicinal treatment, followed by bark and entire plant. Decoction is the most used method of preparation with a percentage of $51.60 \%$. It is followed by infusions (13.07\%), fumigation (12.40\%), poultice $(10.45 \%)$, maceration $(4.58 \%)$, inhalation $(3.90 \%)$, dusting (2.60\%) and drops (1.40\%) (Figure 3).

Among the medicinal plants collected, a majority are sourced from the littoral forest of Agnalazaha while the cultivated fields, weedy disturbed areas, marshes, savannah, savoka (fallow fields), and river follow up (Table 3).

\section{Discussion}

Our focus on the use of medicinal plants by women of Mahabo-Mananivo reinforced the notion that female

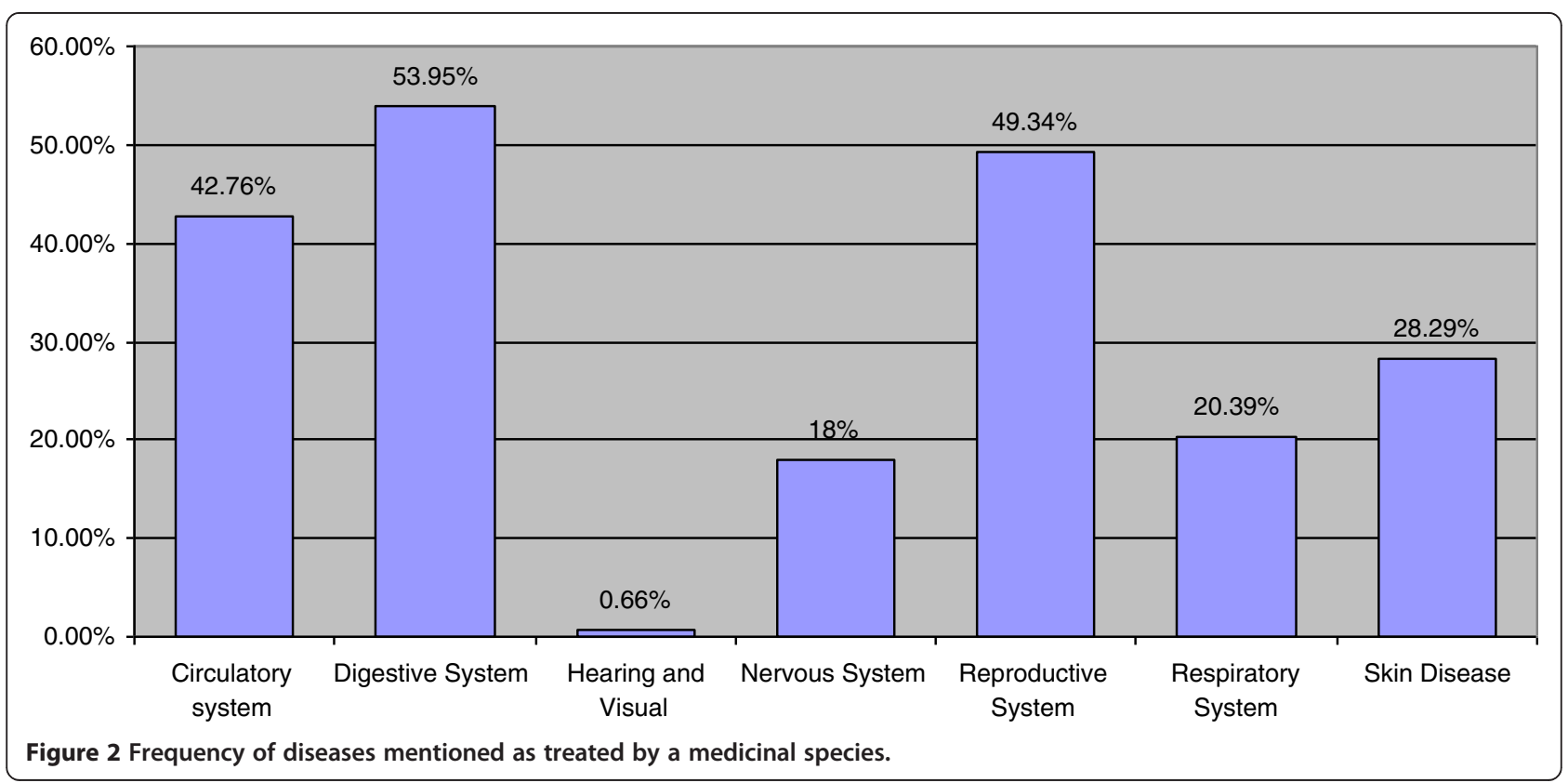






a. Frequency of plant part used

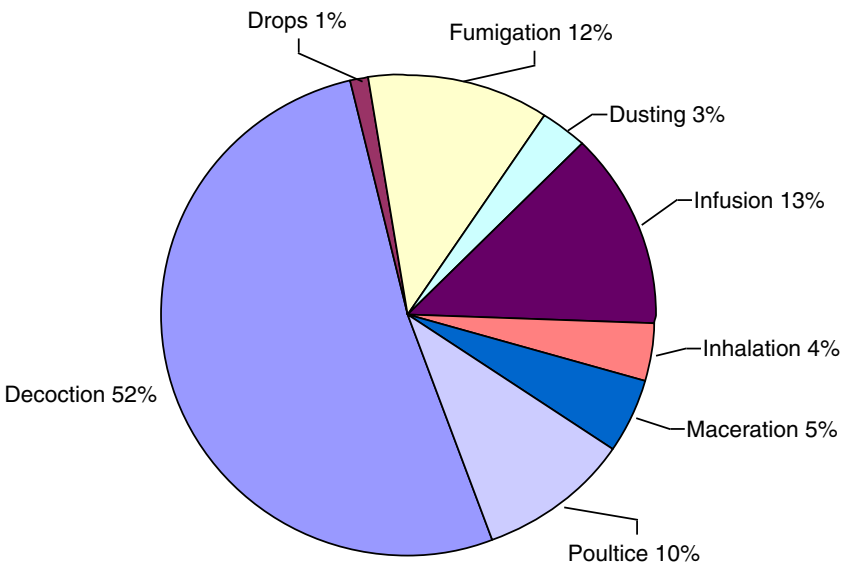

b. Frequency of method of administration

Figure 3 Distribution of type of plant part used (a.) and method of medicinal administration. (b.) Percentage of citations for plant parts used and method of administration for medicinal plant treatment as reported during the interviews. Some plants are noted as having multiple medicinal uses with multiple parts of plants utilized.

caregivers are the first line of health care in many Malagasy homes. We found that traditionally, men collect the medicinal plants while women were mostly responsible for the drying, storage and preparation of the plant to take care of the family members. Reproductive, prenatal and postpartum health were the most frequently cited use for medicinal plants in women's health, a trend seen worldwide [26], however, the women's pharmacopeia was not limited to reproductive and childbirth care and many medicinal species from Agnalazaha Forest are used to treat multiple diseases. We found eight native species that were very well known, and were used to treat multiple diseases. Voacanga thouarsii is used during childbirth and for the treatment of gonorrhea, syphilis, mycosis, wounds, hypertension and is also used for the care of the digestive tract and stomach ulcers. Cinnamosma madagascariensis treats dental decay and general oral care, malaria, and for care of complications after childbirth. Olax emirnensis is used during childbirth, and to treat malaria, hepatitis, epilepsy, dysentery, fatigue, and thought to have magical properties and to provide protection against witchcraft. Syzygium emirnense is used in childbirth, diarrhea, dental disease, and scabies. Nepenthes madagascariensis is used during childbirth, and for treatment of malaria, filariasis, ear infections, syphilis, and gonorrhea. Phyllarthron madagascariense is taken to support breastfeeding, to treat malaria and combat fatigue. Suregada boiviniana helps to evacuate the placenta and treat epilepsy, dysentery, and malaria. Asteropeia micraster also helps to evacuate the placenta and treat diarrhea, fatigue and mumps. Our study found that many of the medicinal species sourced from Agnalazaha Forest were also utilized for other daily living needs. Native medicinal species may also be used as timber, construction materials, and firewood. Conservation concerns mostly lie in the overuse of these valuable daily living species. Conversations with community members highlighted the concern and interest they had for protecting the natural resource of Agnalazaha Forest while ensuring the forest could still be used. It is our goal that through careful ethnobotanical studies of the modern use of Agnalazaha Forest, we can help the community of Mahabo-Mananivo understand their forest use and establish community driven sustainable conservation plans.

\section{Conclusions}

This study highlighted the diversity of medicinal plants used by women and female healers in the Commune of Mahabo-Mananivo. From the perspective of plant diversity,

Table 3 Frequency of localities where medicinal plants are sourced near and around Agnalazaha Forest

\begin{tabular}{|c|c|c|c|c|c|c|c|}
\hline \multicolumn{8}{|c|}{ Sampled locations } \\
\hline & Forest & Marsh & Savanna & Savoka & River & Cultivated & Disturbed areas \\
\hline Frequency (\%) & 40 & 11 & 7 & 4 & 4 & 20 & 14 \\
\hline
\end{tabular}


152 species of medicinal plants in 134 genera and 79 families were identified during the ethnobotanical surveys. First, there is widespread use of medicinal plants that affect the digestive, reproductive and circulatory system. The eight native species widely used are Cinnamosma madagascariensis, Voacanga thouarsii, Nepenthes madagascariensis, Syzigium emirnense, Olax emirnensis, Phyllarthron madagarascariensis, Suregada boiviana, and Asteropeia micraster. This work is only the beginning of a comprehensive study on the ethnobotany of medicinal plants utilized by the community Mahabo-Mananivo from the Agnalazaha Forest. Further studies encompassing ecophysiological, pharmacological and ecological studies are necessary to build a more complete picture on how these rare and compelling littoral forests are used. By documenting the use littoral forest species, we hope to add to the value of these rare forests but also highlight the importance of biodiversity on the health and wellbeing of a community.

\section{Additional file}

Additional file 1: Ethnobotanical questionnaire. PDF of the

questionnaire used during ethnobotanical interviews.

\section{Competing interests}

The authors report no competing interests.

\section{Authors' contributions}

All authors participated in the design of the study and conducted fieldwork. MR analyzed the data. MR and ARK wrote the manuscript. All authors read and approved the final manuscript.

\section{Acknowledgements}

We extend our thanks to the Missouri Botanical Garden and the Plant Biology and Ecology Department for their cooperation which allowed us to carry out research work. We thank our supervisors for their valuable advice, encouragement and methodological guidelines that have allowed us to push this work forward.

We also thank the people in Mahabo-Mananivo, particularly the women and female healers and local guides for graciously providing us with their time and invaluable information.

\section{Author details}

${ }^{1}$ Department of Plant Biology and Ecology, Faculty of Science, University of Antananarivo, BP 566, Antananarivo 101, Madagascar. ${ }^{2}$ Madagascar Research and Conservation Program, Missouri Botanical Garden, BP 3391, Antananarivo 101, Madagascar. ${ }^{3}$ William L. Brown Center, Missouri Botanical Garden, PO Box 299, St. Louis, MO 63166-0299, USA.

Received: 23 April 2013 Accepted: 30 October 2013 Published: 4 November 2013

\section{References}

1. Smith-Hall C, Overgaard Larsen H, Pouliot M: People, plants and health: A conceptual framework for assessing changes in medicinal plant consumption. J Ethnobiol Ethnomed 2012, 8:43.

2. Fabricant DS, Farnsworth NR: The value of plants used in traditional medicine for drug discovery. Environ Health Perspect 2001, 109:69-75.

3. Cunningham AB: African medicinal plants: setting priorities at the interface between conservation and primary health care. Paris:UNESCO: People and Plants Working Paper; 1993

4. Tabuti JR, Lye KA, Dhillion SS: Traditional herbal drugs of Bulamogi, Uganda: plants, use and administration. J Ethnopharmacol 2003, 88(1):19-44.
5. Wilmé L, Goodman SM, Ganzhorn JU: Biogeographic evolution of Madagascar's microendemic biota. Science 2006, 312:1063-1065.

6. Catalogue of the Vascular Plants of Madagascar. [http://www.efloras.org/ madagascar]

7. Schatz GE: Generic tree Flora of Madagascar. London: Royal Botanical Garden: Kew Press; 2001

8. Green GM, Sussman RW: Deforestation history of the eastern rainforests of Madagascar from satellite images. Science 1990, 248:212-215.

9. Alves RRN, Rosa IML: Biodiversity, traditional medicine and public health: where do they meet? J Ethnobiol Ethnomed 2007, 3:14.

10. Novy JW: Medicinal plants of the eastern region of Madagascar. J Ethnopharmacol 1997, 55:119-126.

11. Anyinam C: Ecology and ethnomedicine: exploring links between current environmental crisis and indigenous medical practices. Soc Sci Med 1995, 40(3):321-329.

12. Bollen A, Donati G: Phenology of the littoral forest of Sainte Luce, Southeastern Madagascar. Biotropica 2005, 37(1):32-43.

13. Consiglio T, Schatz G, McPherson G, Lowry PP, Rabenantoandro J, Rogers ZS, Rabevohitra R, Rabehevitra D: Deforestation and plant diversity of Madagascar's littoral forests. Conserv Biol 2006, 20(6):1799-1803.

14. Ludovic R, Rakotoarivony F, Birkinshaw C, Randrianasolo A: Stratégie de conservation communautaire de la forêt d'Agnalazaha. Antananarivo: Missouri Botanical Garden; 2005.

15. Randrianarivelojosia M, Rasidimanana VT, Rabarison $H$, Cheplogoi PK, Ratsimbason M, Mulholland DA, Mauclère P: Plants traditionally prescribed to treat tazo (malaria) in the eastern region of Madagascar. Malar J 2003, 2:25.

16. Wayland C: Gendering local knowledge: medicinal plant Use and primary health care in the Amazon. Med Anthropol Q 2011, 15(2):171-188.

17. Donque G: Contribution géographique à l'étude du climat de Madagascar. Antananarivo: Nouvelle Imprimerie des Arts graphiques; 1975.

18. Koechlin J, Guillaumet JL, Morat P: Flore et végétation de Madagascar. Vaduz, Liechtenstein: J. Cramer; 1974.

19. Andriamaharoa $\mathrm{H}$ : Inventaire des lémuriens dans la forêt d'Agnalazaha Mananivo, Rapport de consultance. Antananarivo: Missouri Botanical Garden; 2004.

20. IUCN Standards and Petitions Subcommittee: Guidelines for Using the IUCN Red List Categories and Criteria. Version 10, Prepared by the Standards and Petitions Subcommittee. Gland, Switzerland: Standards and Petitions Subcommittee; 2013.

21. Tianafidy J: Rapport dinventaire des micro-mammifères dans la foret de Mahabo Mananivo, Rapport de consultance. Antananarivo: Missouri Botanical Garden; 2004.

22. Lyon L, Hardesty L: Quantifying medicinal plant knowledge among non-specialist antanosy villagers in Southern Madagascar. Econ Bot 2012, $66: 1-11$.

23. Martin GJ: Ethnobotany: A Methods Manual. London: Chapman and Hill; 1995.

24. Alexiades MN, Sheldon JW: Selected Guidelines for Ethnobotanical Research: A Field Manual. Bronx, N.Y., U.S.A: New York Botanical Garden; 1996.

25. Gurib-Fakim A, Brendler T: Medicinal and Aromatic Plants of the Indian Ocean Islands. Stuttgart: medpharm Scientific Publishers; 2004.

26. Bussmann RW, Glenn A: Medicinal plants used in Northern Peru for reproductive problems and female health. J Ethnobiol Ethnomed 2010, 6:30.

doi:10.1186/1746-4269-9-73

Cite this article as: Razafindraibe et al:: Medicinal plants used by women from Agnalazaha littoral forest (Southeastern Madagascar). Journal of Ethnobiology and Ethnomedicine 2013 9:73. 